\title{
Periodontal treatment does not result in detectable platelet activation in vivo
}

\author{
Markus Laky ${ }^{1}$ - Isabella Anscheringer ${ }^{2} \cdot$ Lukas Wolschner $^{1} \cdot$ Stefan Heber $^{3} \cdot$ Hady Haririan $^{1} \cdot$ Xiaohui Rausch-Fan $^{1}$. \\ Ivo Volf ${ }^{3}$. Andreas Moritz ${ }^{1} \cdot$ Alice Assinger $^{2}$ (I)
}

Received: 22 March 2019 / Accepted: 5 August 2019/Published online: 29 August 2019

(C) The Author(s) 2019

\begin{abstract}
Objectives Periodontitis is associated with systemic inflammation, elevated platelet activation and enhanced risk for cardiovascular diseases, while periodontal treatment reduces tissue inflammation and shows desirable effects on the oral biofilm and dental health. However, subgingival debridement during conservative treatment can lead to local trauma and transient bacteraemia, which might affect cardiovascular risk in these patients. Therefore, we investigated the effect of periodontal treatment on systemic platelet activation.

Materials and methods In a prospective therapeutic trial, 26 patients underwent periodontal treatment and patient blood was analysed immediately before and immediately after intervention for platelet activation markers (flow cytometric analysis of Pselectin, CD63 and CD40L surface expression, integrin $\alpha \mathrm{IIb} \beta 3$ activation and fibrinogen binding, intra-platelet reactive oxygen species production, platelet-leukocyte aggregate formation and intra-platelet vasodilator-stimulated phosphoprotein phosphorylation) in response to adenosine diphosphate (ADP).

Results The present study shows that basal platelet activation levels remain largely unaltered in response to periodontal treatment. We also did not observe significant changes in platelet reactivity in response to different concentrations of platelet agonist ADP. Conclusion Subgingival debridement does not result in relevantly elevated platelet activation. Thus, augmented platelet activation seems unlikely to be a causative triggering factor that increases the short-term risk for platelet-mediated thrombotic events in response to subgingival debridement.

Clinical relevance Subgingival debridement is a safe procedure and does not increase the short-term risk for platelet-mediated thrombotic events.
\end{abstract}

Keywords Periodontitis $\cdot$ Periodontal treatment $\cdot$ Platelet activation $\cdot$ Cardiovascular disease

\section{Introduction}

Periodontitis, a chronic inflammatory disease of the toothsurrounding tissues, has been associated with systemic inflammation and elevated platelet activation. Thus, it is recognised

Alice Assinger

alice.assinger@meduniwien.ac.at

1 Division of Conservative Dentistry and Periodontology, School of Dentistry, Medical University of Vienna, Vienna, Austria

2 Department for Vascular Biology and Thrombosis Research, Center for Physiology and Pharmacology, Medical University of Vienna, Schwarzspanierstraße 17, Vienna 1090, Austria

3 Institute of Physiology, Center for Physiology and Pharmacology, Medical University of Vienna, Vienna, Austria as an important underlying cause to boost inflammatory mechanisms which lead to the occurrence of cardiovascular diseases (CVD) [1,2]. This makes early treatment of periodontitis an important factor to improve oral health.

The basis of periodontal therapy is anti-infective non-surgical treatment with the objective of controlling the oral biofilm and reducing probing pocket depths (PPD) [3]. Dental plaque and calculus are removed from tooth surfaces with the combined use of manual curettes/scalers and/or powered sonic or ultrasonic cleaning devices [4]. Non-surgical therapy leads to a reduction of tissue inflammation and subsequently to lower PPD and an improved clinical attachment level [5].

More and more evidence emerges that periodontal treatment improves not only oral health but also general health. The achieved benefits in oral health due to periodontal treatment were associated with improvement in endothelial 
function [6] and modulation of periodontitis-associated platelet activation [7].

However, intensive periodontal treatment also leads to local trauma and transient bacteraemia, resulting in acute, shortterm systemic inflammation and endothelial dysfunction [6]. It is currently unknown if subgingival debridement also results in systemic platelet activation and therefore might bear a short-term risk for adverse thrombotic events in patients undergoing this treatment.

Therefore, it was the aim of this study to unravel if subgingival debridement causes systemic platelet activation to better estimate the short-term risk for thrombotic events in patients undergoing periodontal therapy.

\section{Material and methods}

\section{Study design}

Newly diagnosed patients with periodontitis $(n=26)$ presenting to the School of Dentistry, Medical University of Vienna, for periodontal treatment were recruited after initial periodontal screening (inclusion criteria: understanding of the study requirements, at least one interproximal site with a probing depth $\geq 5 \mathrm{~mm}$ and a loss of attachment at $\geq 2$ interproximal sites $\geq 5 \mathrm{~mm}$; exclusion criteria: all systemic diseases, pregnancy or breast feeding, antibiotics in the last 3 months and medication which affects platelets for at least 3 weeks before blood donation). They underwent a baseline periodontal examination and blood testing and full medical and dental histories were collected. Written informed consent was obtained from all study participants before study entry. The Ethics Committee of the Medical University of Vienna approved the study protocol (1656/2014), and the trial was conducted in accordance with the Declaration of Helsinki. The trial has been registered in the ISRCTN registry (\#ISRCTN17035727), and the study uses the treatment group of a larger clinical trial that investigated short-term and long-term impact of subgingival debridement on platelet function and activation for analysis [7]. Patients donated blood before (time point PRE, $0 \mathrm{~h}$ ) and immediately after intensive periodontal treatment (time point POST, $1-2 \mathrm{~h}$ ) at the first treatment session for subgingival debridement.

\section{Periodontal examination and therapy}

Clinical attachment level (CAL), comprising both periodontal probing depth and recession of the gingival margin relative to the cementoenamel junction at six sites per tooth, was examined at baseline with a CP 12 periodontal probe (Hu-Friedy, Frankfurt, Germany). The presence or absence of supragingival dental plaque and gingival bleeding on probing was also recorded. Intensive periodontal treatment was performed by subgingival debridement with curettes and sonic instruments (Sonicflex, KAVO, Biberach, Germany) generally in two to four treatment sessions after the initial examination with a time frame of 1 week or shorter between the sessions. Each session of subgingival debridement lasted for $1-$ $2 \mathrm{~h}$.

\section{Blood collection and platelet function tests}

Venous blood was drawn by the antecubital vein with a $20 \mathrm{G}$ needle and anticoagulated with $3.8 \%$ sodium citrate or ethylenediaminetetraacetic acid (EDTA). Blood count was determined with a Sysmex XP-300 ${ }^{\mathrm{TM}}$ (Sysmex, Vienna, Austria) using EDTA blood. Platelet-rich plasma (PRP) was obtained by centrifugation of citrated blood at $125 \times \mathrm{g}$ for $20 \mathrm{~min}$.

All determinations were performed in duplicate. Platelet function tests were carried out to quantify platelet activation levels without any stimulus (referred to as basal) and to estimate their tendency to become activated by a physiologically relevant stimulus to test platelet reactivity. For measurement of basal platelet activation, phosphate-buffered saline (PBS) was applied instead of agonists. For assessment of platelet reactivity, platelet agonist adenosine diphosphate (ADP, Sigma Aldrich, Vienna, Austria) was incubated with platelets at different concentrations (final concentrations: 2.5, 5 and $50 \mu \mathrm{M}$ ) for $10 \mathrm{~min}$.

Surface expression of P-selectin (CD62P) in unstimulated PRP (basal) represented the primary study outcome. Additional platelet activation markers as well as platelet reactivity in response to ADP stimulation were determined as secondary outcome parameters. Platelets were analysed by flow cytometry (Accuri C6, BD Biosciences, Franklin Lakes, NJ, USA) with analysis software (Accuri C6 software, BD Biosciences) as described previously [7].

\section{Statistical analyses}

This study represents an exploratory analysis of data acquired in a recent randomised controlled trial [7] with an a priori determined sample size of 26 per group, and no additional sample size calculation was performed for the subgroup analysis. In the present study, only data of the treatment group $(n=26)$ were analysed. Based on the exploratory character of this study, no adjustment for multiplicity was performed, and results need to be interpreted accordingly. Platelet activation markers were analysed using mixed linear models with two fixed within-subjects factors ('time point' with the levels 'PRE' and 'POST', 'ADP concentration' with the four concentrations as levels) and patients as levels of a random factor. The Akaike information criterion was used to determine an adequate covariance structure. In case of a significant 'time point' $\times$ 'ADP concentration' interaction, contrasts were used to estimate the differences between time points separately at 
Table 1 Patient periodontal data: periodontal indices of the study participants; $P P D$ periodontal probing depth, $C A L$ clinical attachment level, PESA periodontal epithelial surface area, PISA periodontal inflamed surface area

\begin{tabular}{ll}
\hline & $n=26$ \\
\hline Number of teeth & $27.5(26-29)$ \\
Plaque index, \% & $38.5 \pm 19.5$ \\
Bleeding on probing, \% & $19.6(9.4-29.7)$ \\
PPD $\geq 6 \mathrm{~mm}$, number of sites per patient & $17.5(9-29)$ \\
PPD $\geq 6 \mathrm{~mm}, \%$ & $10.4(6.0-18.5)$ \\
$\mathrm{CAL} \geq 6 \mathrm{~mm}$, number of sites per patient & $27.5(13-40)$ \\
$\mathrm{CAL} \geq 4 \mathrm{~mm}$, number of sites per patient & $82(55-104)$ \\
PESA, mm & $2060 \pm 523$ \\
PISA, $\mathrm{mm}^{2}$ & $543.4 \pm 425$ \\
\hline
\end{tabular}

each ADP concentration. Otherwise, the non-significant interaction term was dropped from the model and the main effect of the time point was used as estimate applicable to all ADP concentrations. For the analysis of VASP phosphorylation, the within-subjects factor PGI (levels: basal, PGI) was used instead of the factor ADP concentration. Right-skewed data were $\log _{10}$ transformed prior to analysis. Statistical analysis was performed with IBM SPSS Statistics 25 . Only two-sided tests were used. $P$ values $\leq 0.05$ were considered significant.

Graphical representation Baseline and post-treatment data of each group are presented as boxplots indicating median, 25th and 75 th percentile, minimum and maximum. Platelet reactivity data (i.e. acquired in the presence of ADP or PGI) are shown as mean \pm SEM.

\section{Results}

We enrolled 26 patients with periodontitis with a median age of 45.5 (25-62) years, $38.5 \%$ were female and the medium body mass index was $23.6(17.9-37.4)$ in this study. More than half of the patients $(61.5 \%)$ were smokers. All patients showed normal blood counts. Mean bleeding on probing (BoP) of patients was at $19.6 \%$. Mean $\mathrm{CAL} \geq 6 \mathrm{~mm}$ was at 27.5 and $\mathrm{CAL} \geq 4 \mathrm{~mm}$ at 82 numbers of sites per patient. Mean PPD $\geq 6 \mathrm{~mm}$ showed 17.5 numbers of sites per patient. The mean periodontal epithelial surface area (PESA) of the patients was $2060 \pm 523 \mathrm{~mm}^{2}$, and the mean periodontal inflamed surface area (PISA) was $543 \pm 425.5 \mathrm{~mm}^{2}$ [8]. The mean periodontal probing depth was $3.55 \pm 0.6 \mathrm{~mm}$. According to the 2018 classification of periodontal diseases and conditions [9], $84.6 \%$ of the included patients had periodontal disease of stage III and $15.4 \%$ of stage IV. For periodontitis progression, $42.3 \%$ were assigned a grade B and $57.7 \%$ a grade $\mathrm{C}$ (Table 1$)$.
No significant changes of platelet activation markers before and immediately after periodontal treatment could be observed (Fig. 1). Only CD40L surface expression was mildly but significantly elevated (Fig. 1c). To evaluate the systemic impact of this effect, we also determined soluble CD40L plasma levels in these patients but did not find significant differences between the two groups (data not shown). When we analysed the amount of reticulated platelets, we saw no difference before and after treatment (Fig. 1i). From these data, we conclude that no systemic platelet activation occurs in response to periodontal treatment.

We then investigated if periodontal treatment affects platelet reactivity in response to platelet agonist ADP. As depicted in Fig. 2, platelet activation in response to different concentrations of ADP did not show significantly enhanced platelet responses. On the contrary, in response to ADP, P-selectin surface expression of platelets was significantly reduced (Fig. 2a) and we further observed a reduction in integrin $\alpha \operatorname{IIb} \beta 3$ activation at moderate and high concentrations of ADP (Fig. 2e). This implies that even if platelets do become activated locally during periodontal treatment, no systemic platelet activation occurred.

\section{Discussion}

In this study, we analysed systemic platelet activation in response to periodontal treatment by utilising a number of different platelet activation markers, including CD62P, CD63 and CD40L. CD62P is part of the platelet $\alpha$ granule membrane, which fuses with the platelet membrane upon exocytosis, while CD63 represents a marker for dense granules and lysosomes. Also CD40L translocates to the platelet surface upon activation [10]. Therefore, these markers were investigated to determine platelet degranulation. However, in our experimental setting, we did not observe changes in CP62P and CD63 surface expression in response to subgingival debridement. CD40L was the only marker that mildly but significantly increased, while plasma levels of soluble CD40L remained unchanged. Platelets represent the main source of soluble CD40L, and elevated plasma levels of soluble CD40L are found in periodontitis patients [11]. Platelet CD40L is known to induce inflammatory responses in the endothelium [12], to initiate the formation of ROS and inhibition of nitric oxide production [13] and to foster the release of chemokines and the expression of several adhesion receptors as well as tissue factors [14]. Further, it plays a role in the progression of plaque formation, macrophage infiltration into the plaque and $\mathrm{T}$ cell homeostasis in the blood and spleen [15]. Since CD40L surface expression was only marginally increased and soluble 
a

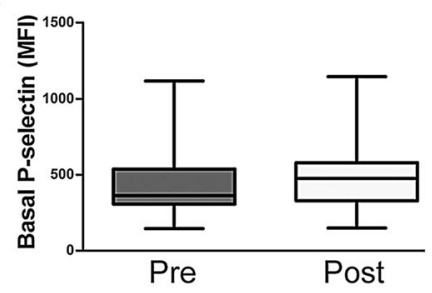

d

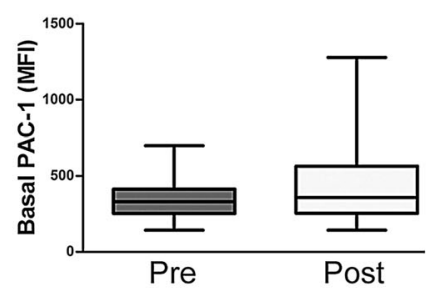

g

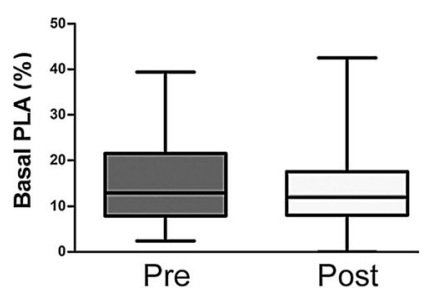

b

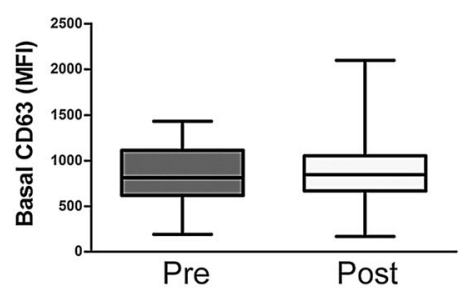

e

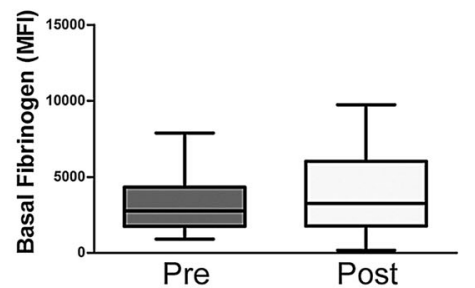

$\mathrm{h}$

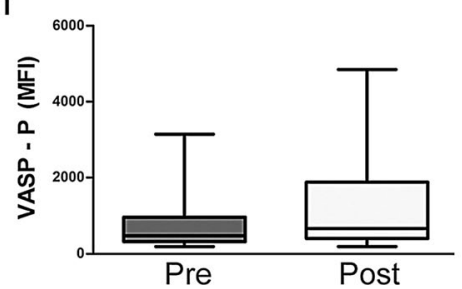

C

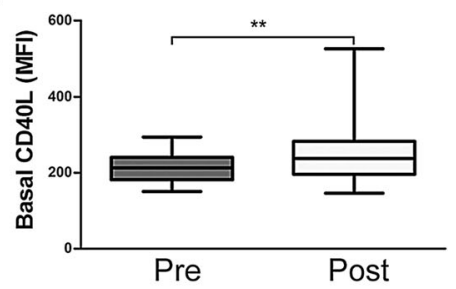

$f$

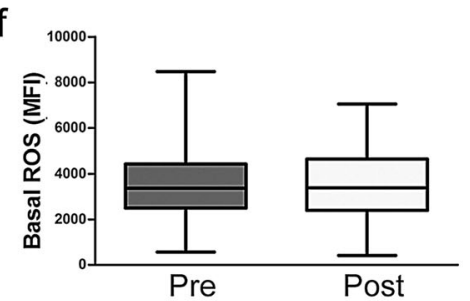

i

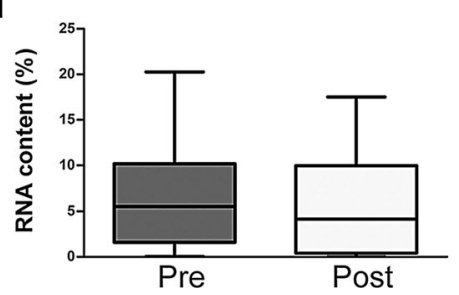

Fig. 1 Effects of periodontal treatment on platelet function. Platelet activation state was determined by surface expression of P-selectin (a), surface expression of CD63 (b), surface expression of CD40L (c), binding of PAC-1 antibody, which represents GPIIb/IIIa activation (d), fibrinogen binding (e), intra-platelet reactive oxygen species (ROS) generation (f), platelet-leukocyte aggregate (PLA) formation (g), intra-platelet vasodilator-stimulated phosphoprotein (VASP) phosphorylation (h) and RNA positive platelets (i); dark grey, before periodontal treatment (PRE); light grey, immediately after periodontal treatment (POST), $n=26$

reactive than older platelets and promote platelet aggregate formation [20] and are indicative for a higher platelet turnover. However, no impact of subgingival debridement on platelet RNA content could be observed in our study. Tonetti et al. [6] and Eickholz et al. [21] found marked systemic effects after subgingival debridement. Although the severity of our study population is comparable, as Tonetti et al. reported a mean number of $84 \pm 26$ periodontal lesions per patient and our patients show a mean number of $82 \pm 33$ periodontal lesions per patient, Eickholz et al. reported a mean periodontal probing depth of $3.5 \pm 0.7 \mathrm{~mm}$ and in our treatment group we found a mean probing depth of $3.55 \pm 0.6 \mathrm{~mm}$; the difference in the outcomes might be attributed to the timing of the subgingival debridement. In our study, subgingival debridement was completed in 2-4 sessions with several days in between. Blood was taken after the debridement of only 1-2 quadrants whereas Eickholz et al. performed subgingival debridement in 1-2 visits at two consecutive days and Tonetti et al. made a full mouth intensive subgingival debridement. The extent of the subgingival debridement might be an explanation for the different findings.

Our result suggests that even if platelets become locally activated by the periodontal treatment, systemically no increase in platelet activation can be detected. We previously observed a similar phenomenon in healthy volunteers that 
Fig. 2 Effects of periodontal treatment on platelet response to different concentrations of ADP. Platelet response to ADP was measured after stimulation with 2.5, 5 and $50 \mu \mathrm{M}$ ADP and responses monitored by surface expression of P-selectin (a), surface expression of CD63 (b), surface expression of CD40L (c), platelet-leukocyte aggregate (PLA) formation (d), binding of PAC-1 antibody, which represents GPIIb/IIIa activation (e),

fibrinogen binding (f) and intra-platelet vasodilator-stimulated phosphoprotein (VASP) phosphorylation (g); dark grey, before periodontal treatment (PRE); light grey, immediately after periodontal treatment (POST), $n=26$ a

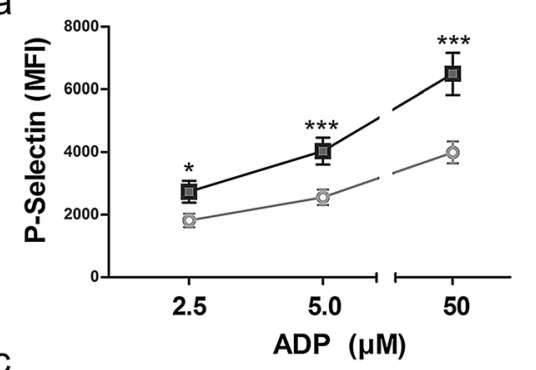

C
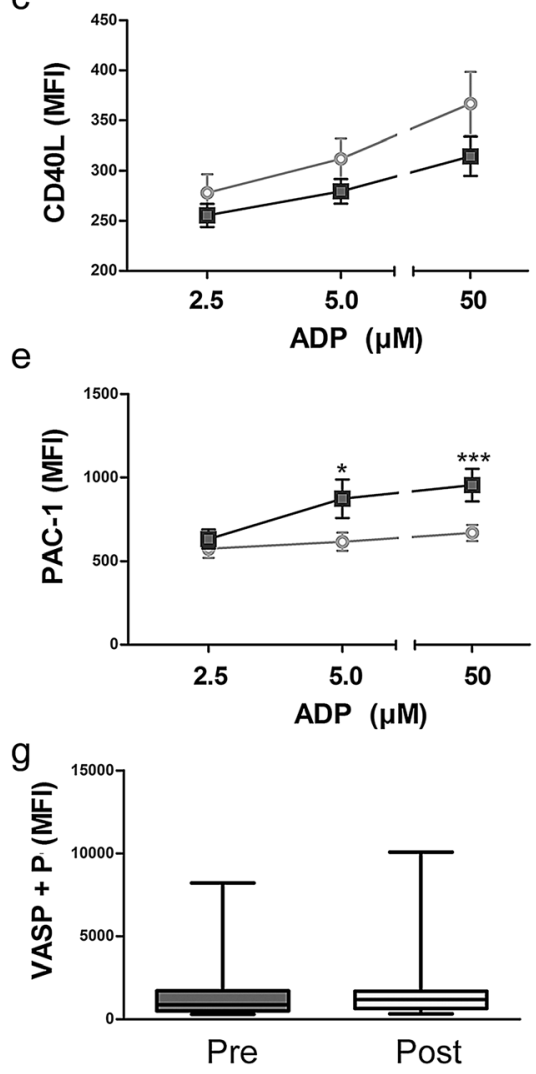

b
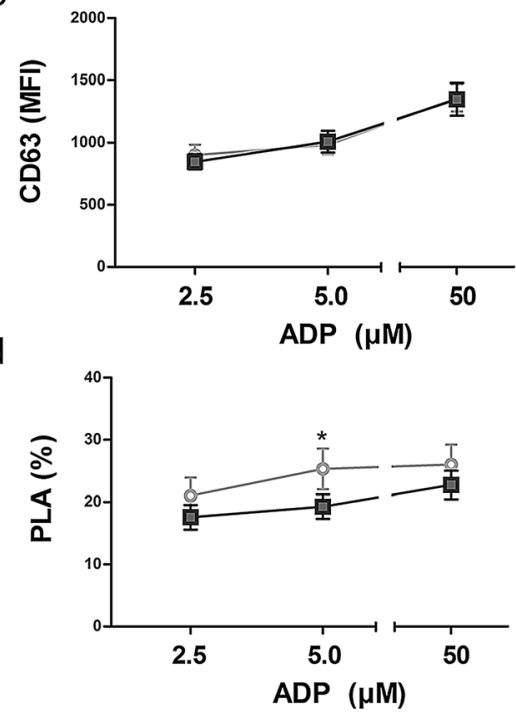

f

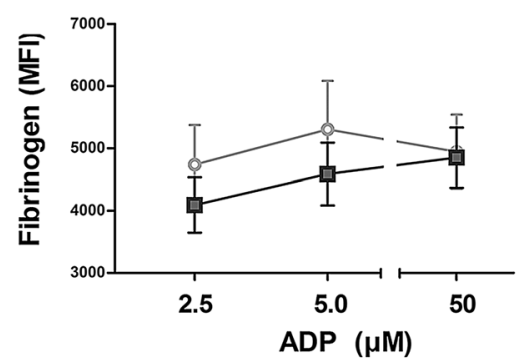

were challenged with an endotoxin stimulus [22]. While platelets did locally respond to the toxin, systemically no increase in platelet activation occurred [22], suggesting that activated platelets may get scavenged and are therefore no longer detected in the circulation.

Of note, all patients were followed up for a period of 3 months and platelet activation markers were then investigated again and compared with a control cohort. We found that periodontal treatment was able to limit platelet activation in patients with periodontitis [7], indicating beneficial systemic effects of periodontal therapy.

Our investigation has certain limitations. Firstly, the number of individuals included in this analysis is relatively small and therefore the findings should be interpreted with caution. Further, we only investigated a single-very early-time point. It is possible that platelet activation and/or reactivity increase at later time points, where also endothelial cell and leukocyte activation were detected. It is known that intensive periodontal therapy induces a moderate acute systemic inflammatory response associated with endothelial cell activation and mildly increased plasma cytokine levels [6, 21, 23, 24]. However, this implies that platelets get only indirectly activated by other cells and not due to the treatment as such. Additionally, activated platelets might get scavenged and therefore might not be longer detectable in the circulation as observed in previous studies in response to inflammatory stimuli [22]. In this study, we did not include a control group, since blood was taken immediately before and immediately after gingival debridement. So both blood samples were taken within less than $2 \mathrm{~h}$, and the first time point served as a baseline measurement for the second time point. It is known that platelet activation is enhanced in patients with periodontitis $[11,25,26]$ but no further enhancement could be observed upon subgingival debridement.

From our data, we conclude that subgingival debridement does not result in elevated platelet activation and therefore 
does not seem to increase the immediate platelet-related adverse events.

Acknowledgements The authors would like to thank Angela Knöbl and Susanne Humpeler for their technical support.

Funding Information Open access funding provided by Medical University of Vienna. The study was financed by the Anniversary Fund of the Austrian National Bank (OeNB grant no. 15961), Austrian Science Fund (FWF P-24978) and the Medical Scientific Fund of the Mayor of the City of Vienna (no. 15150).

\section{Compliance with ethical standards}

Conflict of interest The authors declare that they have no conflict of interest.

Ethical approval All procedures performed in studies involving human participants were in accordance with the ethical standards of the institutional and/or national research committee and with the 1964 Helsinki declaration and its later amendments or comparable ethical standards.

Informed consent Written informed consent was obtained from all subjects included in this study.

Open Access This article is distributed under the terms of the Creative Commons Attribution 4.0 International License (http:// creativecommons.org/licenses/by/4.0/), which permits unrestricted use, distribution, and reproduction in any medium, provided you give appropriate credit to the original author(s) and the source, provide a link to the Creative Commons license, and indicate if changes were made.

\section{References}

1. Humphrey LL, Fu R, Buckley DI, Freeman M, Helfand M (2008) Periodontal disease and coronary heart disease incidence: a systematic review and meta-analysis. J Gen Intern Med 23(12):20792086. https://doi.org/10.1007/s11606-008-0787-6

2. Bahekar AA, Singh S, Saha S, Molnar J, Arora R (2007) The prevalence and incidence of coronary heart disease is significantly increased in periodontitis: a meta-analysis. Am Heart J 154(5):830 837. https://doi.org/10.1016/j.ahj.2007.06.037

3. Pihlstrom BL, Michalowicz BS, Johnson NW (2005) Periodontal diseases. Lancet 366(9499):1809-1820. https://doi.org/10.1016/ S0140-6736(05)67728-8

4. Heitz-Mayfield LJ, Lang NP (2013) Surgical and nonsurgical periodontal therapy. Learned and unlearned concepts. Periodontol 62(1):218-231. https://doi.org/10.1111/prd.12008

5. Drisko CH (2001) Nonsurgical periodontal therapy. Periodontol 25: 77-88

6. Tonetti MS, D'Aiuto F, Nibali L, Donald A, Storry C, Parkar M, Suvan J, Hingorani AD, Vallance P, Deanfield J (2007) Treatment of periodontitis and endothelial function. N Engl J Med 356(9): 911-920. https://doi.org/10.1056/NEJMoa063186

7. Laky M, Anscheringer I, Wolschner L, Heber S, Haririan H, Schrottmaier WC, Kral-Pointner JB, Salzmann M, Volf I, Moritz A, Assinger A (2018) Periodontal treatment limits platelet activation in patients with periodontitis-a controlled-randomized intervention trial. J Clin Periodontol 45(9):1090-1097. https://doi.org/ $10.1111 /$ jcpe. 12980
8. Nesse W, Abbas F, van der Ploeg I, Spijkervet FK, Dijkstra PU, Vissink A (2008) Periodontal inflamed surface area: quantifying inflammatory burden. J Clin Periodontol 35(8):668-673. https:// doi.org/10.1111/j.1600-051X.2008.01249.x

9. Papapanou PN, Sanz M, Buduneli N, Dietrich T, Feres M, Fine DH, Flemmig TF, Garcia R, Giannobile WV, Graziani F, Greenwell H, Herrera D, Kao RT, Kebschull M, Kinane DF, Kirkwood KL, Kocher T, Kornman KS, Kumar PS, Loos BG, Machtei E, Meng H, Mombelli A, Needleman I, Offenbacher S, Seymour GJ, Teles R, Tonetti MS (2018) Periodontitis: consensus report of workgroup 2 of the 2017 World Workshop on the Classification of Periodontal and Peri-Implant Diseases and Conditions. J Periodontol 89(Suppl 1):S173-S182. https://doi.org/10.1002/JPER.17-0721

10. Hermann A, Rauch BH, Braun M, Schror K, Weber AA (2001) Platelet CD40 ligand (CD40L)-subcellular localization, regulation of expression, and inhibition by clopidogrel. Platelets 12(2):74-82

11. Assinger A, Laky M, Badrnya S, Esfandeyari A, Volf I (2012) Periodontopathogens induce expression of CD40L on human platelets via TLR2 and TLR4. Thromb Res 130(3):e73-e78. https://doi. org/10.1016/j.thromres.2012.04.017

12. Henn V, Slupsky JR, Grafe M, Anagnostopoulos I, Forster R, Muller-Berghaus G, Kroczek RA (1998) CD40 ligand on activated platelets triggers an inflammatory reaction of endothelial cells. Nature 391(6667):591-594. https://doi.org/10.1038/35393

13. Urbich C, Dernbach E, Aicher A, Zeiher AM, Dimmeler S (2002) CD40 ligand inhibits endothelial cell migration by increasing production of endothelial reactive oxygen species. Circulation 106(8): 981-986

14. Slupsky JR, Kalbas M, Willuweit A, Henn V, Kroczek RA, MullerBerghaus G (1998) Activated platelets induce tissue factor expression on human umbilical vein endothelial cells by ligation of CD40. Thromb Haemost 80(6):1008-1014

15. Lievens D, Zernecke A, Seijkens T, Soehnlein O, Beckers L, Munnix IC, Wijnands E, Goossens P, van Kruchten R, Thevissen L, Boon L, Flavell RA, Noelle RJ, Gerdes N, Biessen EA, Daemen MJ, Heemskerk JW, Weber C, Lutgens E (2010) Platelet CD40L mediates thrombotic and inflammatory processes in atherosclerosis. Blood 116(20):4317-4327. https://doi.org/10.1182/blood-201001-261206

16. Bennett JS (2005) Structure and function of the platelet integrin alphaIIbbeta3. J Clin Invest 115(12):3363-3369. https://doi.org/ 10.1172/JCI26989

17. Schrottmaier WC, Kral JB, Badrnya S, Assinger A (2015) Aspirin and P2Y12 inhibitors in platelet-mediated activation of neutrophils and monocytes. Thromb Haemost 114(3):478-489. https://doi.org/ 10.1160/TH14-11-0943

18. Assinger A, Schmid W, Volf I (2010) Decreased VASP phosphorylation in platelets of male and female smokers of young age. Platelets 21(8):596-603. https://doi.org/10.3109/09537104.2010. 505674

19. Fujii T, Shimomura T, Fujimoto TT, Kimura A, Fujimura K (2000) A new approach to detect reticulated platelets stained with thiazole orange in thrombocytopenic patients. Thromb Res 97(6):431-440

20. Armstrong PC, Hoefer T, Knowles RB, Tucker AT, Hayman MA, Ferreira PM, Chan MV, Warner TD (2017) Newly formed reticulated platelets undermine pharmacokinetically short-lived antiplatelet therapies. Arterioscler Thromb Vasc Biol 37(5):949-956. https:// doi.org/10.1161/ATVBAHA.116.308763

21. Eickholz P, Siegelin Y, Scharf S, Schacher B, Oremek GM, SauerEppel H, Schubert R, Wohlfeil M (2013) Non-surgical periodontal therapy decreases serum elastase levels in aggressive but not in chronic periodontitis. J Clin Periodontol 40(4):327-333. https:// doi.org/10.1111/jcpe.12076

22. Schrottmaier WC, Kral JB, Zeitlinger M, Salzmann M, Jilma B, Assinger A (2016) Platelet activation at the onset of human 
endotoxemia is undetectable in vivo. Platelets 27(5):479-483. https://doi.org/10.3109/09537104.2015.1119814

23. D'Aiuto F, Parkar M, Tonetti MS (2007) Acute effects of periodontal therapy on bio-markers of vascular health. J Clin Periodontol 34(2):124-129. https://doi.org/10.1111/j.1600-051X.2006.01037.x

24. Ramich T, Asendorf A, Nickles K, Oremek GM, Schubert R, Nibali L, Wohlfeil M, Eickholz P (2018) Inflammatory serum markers up to 5 years after comprehensive periodontal therapy of aggressive and chronic periodontitis. Clin Oral Investig 22(9):3079-3089. https://doi.org/10.1007/s00784-018-2398-x

25. Laky M, Assinger A, Esfandeyari A, Bertl K, Haririan H, Volf I (2011) Decreased phosphorylation of platelet vasodilator- stimulated phosphoprotein in periodontitis-a role of periodontal pathogens. Thromb Res 128(2):155-160. https://doi.org/10.1016/ j.thromres.2011.02.016

26. Assinger A, Buchberger E, Laky M, Esfandeyari A, Brostjan C, Volf I (2011) Periodontopathogens induce soluble P-selectin release by endothelial cells and platelets. Thromb Res 127(1):e20-e26. https://doi.org/10.1016/j.thromres.2010.10.023

Publisher's note Springer Nature remains neutral with regard to jurisdictional claims in published maps and institutional affiliations. 